\title{
Human disease-related pathways increasing with opportunistic pathogens bloom in intestinal microbiome after vancomycin exposure and restoration with fecal transplantation by simulator of the human intestinal microbial ecosystem (SHIME)
}

Lei Liu

Nankai University

Qing Wang

Nankai University

Hongmei Qi

Nankai University

Ranjit Das

Nankai University

Huai Lin

Nankai University

Jingliang Shi

Nankai University https://orcid.org/0000-0003-0561-9083

Siyi Wang

Nankai University

Jing Yang

Nankai University

Yingang Xue

Nankai University

Daqing Mao

Nankai University School of Medicine

Yi Luo ( $D$ luoy@nankai.edu.cn )

https://orcid.org/0000-0002-6732-7935

\section{Research}

Keywords: Fecal microbiota transplantation (FMT), Human disease-related pathways, Opportunistic pathogen, Simulator of the human intestinal microbial ecosystem (SHIME), Vancomycin 
Posted Date: December 31st, 2019

DOI: https://doi.org/10.21203/rs.2.19821/v1

License: (c) (i) This work is licensed under a Creative Commons Attribution 4.0 International License. Read Full License 


\section{Abstract}

Background: Antibiotics are growing reported to cause human intestinal microbial disorders with increasing abundances of opportunistic pathogens, and fecal microbiota transplantation (FMT) has been confirmed to restore the dysbiosis of gut flora in many kinds of intestinal disease. However, to date, few studies have focused on the bloomed opportunistic pathogens associated human disease-related pathways as well as antibiotic resistance genes (ARGs) after vancomycin exposure, and there is limited information on using FMT for restoration of intestinal microbiome affected by antibiotics.

Methods: This study investigated effects of vancomycin on the opportunistic pathogens, human diseaserelated pathways as well as ARGs in human gut, and the restoration of intestinal microbiome by FMT. Using the simulator of the human intestinal microbial ecosystem (SHIME), two reactors (representing the ascending and descending colon, respectively) were inoculated with human intestinal microbiota from a healthy adult volunteer who did not suffer from gastrointestinal diseases or take any antibiotic in the previous six months. Samples were collected from the reactors at six-time points (before treatment, after seven days of individual $10 \mathrm{mg} / \mathrm{L}, 100 \mathrm{mg} / \mathrm{L}, 1000 \mathrm{mg} / \mathrm{L}$ vancomycin treatment, 14 days following the termination of vancomycin and 14 days following FMT). The intestinal microbiome composition and function were analyzed using 16S rRNA gene sequencing and High-throughput Quantitative PCR (HTqPCR).

Results: Vancomycin treatment substantially increased human disease-related pathways and decreased abundances of ARGs. Besides, the bloomed opportunistic pathogens including Achromobacter, Klebsiella, and Pseudomonas, caused by vancomycin exposure, were positively correlated with human diseaserelated pathways. The microbiota abundance and genes of human disease-related pathways and antibiotic resistance showed a remarkable return towards baseline after FMT, but not for natural recovery.

Conclusions: These findings suggest that impacts of vancomycin on human gut are profound and FMT will be a promising strategy in clinical application that can restore the dysbiosis of gut microbiota, which may be valuable for directing future work.

\section{Background}

The human intestine constitutes primarily a large and diverse microbial habitat that is colonized by many bacteria, as well as archaea, viruses, and eukaryotes [1]. The human intestinal microbiota has been demonstrated to provide numerous essential functions for human health including nutrient supply, modulating the immune function, protection from the infectious pathogens, and metabolism of xenobiotic drugs $[2,3]$. Both human and veterinary antibiotics were detected in the collective gut of the Chinese population through our previous research [4]. The antibiotic therapies have been demonstrated paramount importance in the treatment of numerous diseases caused by bacterial infections since its discovery in the 1940s. Concerns about the use of antibiotics have traditionally focused on the killing of bacterial pathogens; however overuse and misuse of antibiotics may promote the spread of antibiotic- 
resistant bacteria and the antibiotic resistance genes (ARGs) which may limit the treatment efficiency and resulting in chronic and relapsing infections $[5,6]$. Recent studies have found that indiscriminately kill or prevent the growth of both pathogenic and commensal bacteria by antibiotics may cause the dysbiosis of intestinal microbiota, and then will promote pathology, including obesity, asthma and infectious diseases $[7,8]$.

Vancomycin, a glycopeptide antibiotic is mainly active against the gram-positive bacteria and is commonly used to treat the infections produced by multidrug-resistant Staphylococcus aureus and Clostridium difficile. However, researches have reported that the administration of vancomycin would increase the abundance of opportunistic pathogens such as Pseudomonas and Klebsiella in human intestinal microbiota $[9,10]$. Furthermore, few studies have focused on the bloomed opportunistic pathogens associated human disease-related pathways as well as ARGs after vancomycin exposure. These opportunistic pathogens are ubiquitous in the environment and are known to be considered as commensal in the human gut; but the increasing numbers of their population could be a significant risk factor for the infection $[11,12]$. Moreover, these opportunistic pathogens are prominently linked with human diseases such as pneumonia, colitis, cancer, diabetes, neurosurgery, and cardiovascular diseases $[13,14]$.

Previous studies showed that many researchers have investigated influences of vancomycin on the human intestinal microbiota in vivo $[9,10,15]$. However, in vitro experiments are very important for clarifying direct effects of antibiotics on human intestinal microbiota without the disturbances from neurohumoral regulation, the individual differences, dietary habits, and physiological status. The simulator of the human intestinal microbial ecosystem (SHIME) model is known to be a useful tool for in vitro studies as (i) interactions between the microbiota; and (ii) the effects of prebiotics and other compounds on the microbial communities and metabolic activities [16]. However, to the best of our knowledge, there are very few researches that added the antibiotics into this in vitro model [17-20]. Researches mainly focused on the benefit of the mucosal environment, high-fiber diets, probiotic, and propionate-producing consortium in human intestinal microbiota. The effects of antibiotics, including AMX and other antibiotics mixture, vancomycin, and clindamycin, were limited to microbiota composition and metabolite.

Fecal microbiota transplantation (FMT) is the introduction of feces from a healthy donor into the intestinal tract of someone with disrupted microbiota, which has been confirmed superior to oral vancomycin for the treatment of Clostridium difficile infection [21, 22]. And combination of FMT with vancomycin could be a better choice to treat this kind of infection [23, 24]. However, there is limited information on using fecal microbiota transplantation (FMT) for restoration of intestinal microbiome affected by antibiotics. Therefore, it is exciting for us to investigate whether FMT would restore the human intestinal dysbiosis caused by vancomycin exposure.

Since the reasonable dosage of vancomycin for adult human study is about 500 to $1500 \mathrm{mg} \mathrm{day}^{-1}$ and only half volume of adult gut can be simulated in the used SHIME model, here the direct effects of 
$600 \mathrm{mg} \mathrm{day}^{-1}\left(1000 \mathrm{mg} \mathrm{L}^{-1}\right)$ of vancomycin on the composition and human disease-related pathways as well as ARGs of the human gut microbiota were studied $[15,24]$. And $10 \mathrm{mg} \mathrm{L}^{-1}$ and $100 \mathrm{mg} \mathrm{L}^{-1}$ were chosen as lower doses of vancomycin to verify whether these doses of vancomycin would affect human gut microbiota, because the vancomycin agar plate concentration for vancomycin resistant bacteria isolation is between this range according to the CLSI M07-A8 2011 guidelines. In this study, the composition of human intestinal microbiota composition and human disease-related pathways were analyzed by 16S rRNA gene high-throughput sequencing, and the ARGs were quantified by highthroughput quantitative PCR (HT-qPCR). This study achieved a systematical investigation and precisely understanding of the direct effects of vancomycin modulation on the intestinal microbiota and the restoration of FMT using a SHIME model system, which may be valuable for directing future work.

\section{Methods}

\section{Vancomycin treatment and sample collection}

The SHIME model in this study was formed by four double-jacketed reactors designated as the stomach, small intestine, ascending colon, and descending colon, respectively (Fig. S1a). The last two reactors inoculated with a mixture of fecal microbiota collected from a healthy adult volunteer, who did not suffer from gastrointestinal diseases or take any antibiotics in the previous six months, and the differences between individuals may be alleviated by same culture condition [25]. The details of the SHIME system and the startup process are summarized in the Supplementary material.

After two weeks' stabilization and another week's nutritional medium adding, the SHIME sequentially exposed to a gradient of vancomycin (from low to high concentrations) and experienced two weeks' natural recovery and two weeks' FMT. All the samples were collected and analyzed at six-time points: prior to the treatment (C_A, C_D), and after 7 days of each administration of $10 \mathrm{mg} \mathrm{L}^{-1}$ (V10_A, V10_D), $100 \mathrm{mg} \mathrm{L}^{-1}$ (V100_A, V100_D), and $1000 \mathrm{mg} \mathrm{L}^{-1}$ vancomycin (V1000_A, V1000_D), after 14 days of vancomycin discontinuation (NR_A, NR_D), and after 14 days of FMT (FR_A, FR_D), and each sample is a mixture of three samples collected from 7 a.m. to 11 p.m. (Fig. S1b). The samples were stored at $-80^{\circ} \mathrm{C}$ for further analyses.

\section{S rRNA gene sequencing and analysis}

Microbial DNA was extracted from the samples using the E.Z.N.A. stool DNA Kit (Omega, USA) according to the manufacturer's protocols. The V3-V4 region of the bacterial 16S rRNA gene was amplified by polymerase chain reaction (PCR). The raw reads were deposited into the NCBI Sequence Read Archive (SRA) database under the accession number SRR9329997-9330008.

Raw Illumina fastq files were de-multiplexed, quality-filtered, and analyzed using Quantitative Insights Into Microbial Ecology (QIIME) [26]. The 16S rRNA gene sequences were classified taxonomically using the Ribosomal Database Project (RDP) classifier 2.0.1 [27]. To investigate the effects of vancomycin on alpha diversity, the taxon richness (Chao1 index), evenness (Simpson index), and diversity (Shannon 
index) were calculated for all samples. In addition, beta diversity of the microbiota communities at baseline and after antibiotics were portrayed by nonmetric multidimensional scaling (NMDS) and principal coordinate analysis (PCoA) of weighted and unweighted UniFrac distances [28]. Linear discriminant analysis effect size (LEfSe) analysis was performed to determine bacterial taxa that significantly differed between the control and antibiotic group using the Galaxy application tool [29]. Functional predictions of microbial community were performed to visualize the distribution of human disease related pathways in the two parts of colon with different treatment using Phylogenetic Investigation of Communities by Reconstruction of Unobserved States (PICRUSt) [30]. The accuracy of PICRUST for the detection of these more challenging functional groups was good ( $\mathrm{min}$. accuracy $=0.82$ ), suggesting that their inference of gene abundance across various types of functions was reliable, and PICRUSt predictions had high agreement with metagenome sample abundances across all body sites (Spearman $r=0.82, P<0.001$ ). The details of taxonomical classification, LEfSe analysis, and functional predictions were described in the Supplementary material.

\section{High-throughput quantitative PCR (HT-qPCR) and analysis}

HT-qPCR reactions were performed to visualize the variation of ARGs during the treatment using Wafergen SmartChip Real-time PCR system, which was conducted by Anhui MicroAnaly Gene Technologies Co., Ltd (Anhui, China). A total of 120 primer sets were used (Excel S1), including 114 primer sets to target almost all major classes of antibiotic resistance genes (ARGs) found in Chinese human gut microbiota [31], five mobile genetic elements (MGEs) and one 16S rRNA gene. The results were analyzed with SmartChip qPCR Software excluding the wells with multiple melting peaks or amplification efficiency beyond the range (90-110\%) and then screened with conditions that a threshold cycle (CT) must be $<31$ and positive samples should have three replicates simultaneously. The details of HT-qPCR analysis were described in the Supplementary material.

\section{Antibiotic susceptibility test and serum killing resistance assays of pathogens}

The Pathogenic bacterial strains were isolated from the V1000_A sample using Luria-Bertani (LB) agar

plates supplemented with $100 \mathrm{mg} \mathrm{L}^{-1}$ of vancomycin. Genomic DNA of these strains was extracted using a Promega Wizard Genomic DNA Purification Kit (Promega, Madison, USA) according to the manufacturer's protocols. The isolated strains were identified by $16 \mathrm{~S}$ ribosomal DNA sequencing conducted by BGI Tech Solutions (Beijing Liuhe) Co., Ltd (Beijing, China). Nine antibiotic resistance genes from the isolated strains were confirmed using Qualitative PCR with the primers listed in Table S1. MICs of these pathogens were determined according to the CLSI M07-A8 2011 guidelines. The serum-killing assay was performed based on the method described by David and associates [32]. Details of the antibiotic susceptibility test, and the serum killing resistance assays procedures are described in the Supplementary material.

\section{Data analysis}


Correlation between the microbiota and human disease-related pathways or ARGs were analyzed using the Spearman test in $\mathrm{R}$ with the vegan package. Correlations between pairs of variables were considered to be significant at $r>0.6$, and $P$ values were $<0.05$. The Gephi $(V 0.9 .1)$ software was used to visualize the bipartite network graphs using the Force Atlas algorithm.

\section{Results}

\section{Human disease-related pathways and ARGs}

The human disease-related pathways and ARGs in samples from the SHIME model were assessed at different time points, which included samples prior to antibiotic treatment $(C)$, sequential doses $(10,100$, and $1000 \mathrm{mg} \mathrm{L}^{-1}$ ) of antibiotic treatments at seven days interval designated as V10, V100, and V1000, followed by 14 days after termination of vancomycin (NR) and 14 days after FMT (FR). The hierarchy cluster heatmap showed that gene numbers of human disease-related pathways, including drug resistance, cancer, cardiovascular diseases, immune system diseases, infectious diseases, metabolic diseases, and neurosurgery diseases were more abundant in antibiotic exposure groups than that in control group, which was especially apparent in ascending colon (Fig. 1). For instance, gene numbers of bladder cancer, renal cell carcinoma, primary immunodeficiency, type II diabetes mellitus, and prion diseases were nearly 1.3-1.5 times more enriched in the V1000 group than in control. Moreover, gene numbers of these human disease-related pathways still maintained at a higher level (about 1.2-1.3 times enriched than control) after natural recovery and FMT treatment could restore them to baseline level.

The relative abundance of ARGs such as aminoglycoside, beta-lactam, multidrug, and tetracycline resistance genes were lower in vancomycin exposure groups than that in control samples, which was also especially obvious in ascending colon (Fig. 2). For instance, log relative abundance reduction of aac6ie (aminoglycoside) was about $2.9 \mathrm{log}$ units after $1000 \mathrm{mg} \mathrm{L}^{-1}$ vancomycin treatment than in control, and bl2b_tem1 (beta_lactam), ermb (MLSB), and qacedelta1 (multidrug) were about 2.0, 2.3, and $1.5 \log$ units, respectively. Besides, the ARGs such as ant3ia (aminoglycoside), bl1_ec (beta_lactam), ermf (MLSB), mexf (multidrug), tetq (tetracycline), and sul1 (sulfonamide) were not detected after vancomycin treatment. Similarly, these ARGs were unable to return to the baseline level following the natural recovery with about 2.2 to $3.8 \mathrm{log}$ units lower of relative abundance. FMT would restore most of them to some extent (about 1.7 log units lower to 0.6 log units higher).

\section{Microbiota community composit ion and diversity}

In this study, the effects of vancomycin on gut microbial communities' composition were also investigated. Based on the 16S rRNA gene sequence analysis, the most abundant taxonomic groups assigned at the phylum level were Proteobacteria (64.7-98.7\%), Bacteroidetes $(0.1-18.7 \%)$ and Firmicutes (0.5-12.4\%) present in the ascending colon (total of 92.5-99.4\%), followed by Synergistetes, Fusobacteria, and Verrucornicrobia (Fig. S2). However, the most three abundant bacterial phyla in the 
descending colon (total of 79.8-94.8\%) were Proteobacteria (53.6-83.4\%), Bacteroidetes (0.1-23.0\%) and Synergistetes (2.3-10.8\%). Moreover, an obvious decrease of Bacteroidetes (from 17.7-18.7-0.1\%) and Firmicutes (from 5.9-12.4\% to $0.5-0.9 \%$ ), and increase of Proteobacteria (from 68.3-68.9\% to 83.4$98.7 \%$ ) were seen after $1000 \mathrm{mg} \mathrm{L}^{-1}$ vancomycin treatment. The significant changed communities' composition at the phylum level was sustained after natural recovery and returned to baseline after FMT treatment. At the genus level, the antibiotic-treated subjects were shown to be substantially overgrown by Burkholderia (from $0.2-3.2 \%$ to $2.8-17.9 \%$ ) and Achromobacter (from $0.3-0.4 \%$ to $6.3-8.9 \%$ ) with the dose of vancomycin, while the percentage of Pseudomonas was increased in the V10 group (from 6.9$45.5 \%$ to $17.0-53.2 \%$ ) and Klebsiella increased in the V1000 group (from $15.0-39.7 \%$ to $22.3-51.4 \%$ ). Similarly, following the natural recovery period, the gut microbiota was incompletely restored, and the complete recovery observed after FMT. These results were in line with human disease-related pathways and ARGs results.

Meanwhile, fecal microbiota alpha diversity was assessed. The taxon richness (Chao1 index), evenness (Simpson index), and diversity (Shannon index) are shown in Fig. S3a to c. Compared with control sample C_A, a drop in microbiota richness (Chao1), evenness (Simpson), and diversity (Shannon) was observed in sample collected from ascending colon after $1000 \mathrm{mg} \mathrm{L}^{-1}$ of vancomycin treatment; however opposite change was observed in sample V10_A. As shown in Fig. S3, the decreasing of microbiota richness, evenness, and diversity caused by vancomycin treatment restored after two weeks of vancomycin discontinued (NR). Besides, the beta diversity of the microbiota communities and weighted UniFrac distance was affected by antibiotic treatment. As shown in Fig. 3, the samples that collected after vancomycin treatments were differed from the control group in both UniFrac NMDS and PCoA analyses. The beta diversity results also showed that gut microbial composition remained comparable after two weeks of vancomycin discontinued (NR) because these two samples were clustered together with vancomycin treatment groups. Meanwile, after two weeks of FMT treatment, the samples clustered to control group for both UniFrac NMDS and PCoA analyses. Moreover, weighted UniFrac distance between $1000 \mathrm{mg} \mathrm{L}^{-1}$ vancomycin treatment group and control group (V1000 vs. C) were higher than that within the control group (C vs. C), and that between the recovery groups and the control group (NR vs. C, FR vs. C) were slightly higher (Fig. S4).

\section{Bloomed opportunistic pathogens}

The linear discriminant analysis effect size (LEfSe) comparison analysis between the control and antibiotic groups was shown in Fig. 4. LEfSe analysis indicated that vancomycin resulted in significant decreases in several taxa, including the members of Bacteroidetes (Bacteroides, Parabacteroides), Firmicutes (Clostridium, Dialister) and Campylobacter. The changes were accompanied by increases in the relative abundance of Proteobacteria phylum, including Achromobacter (LSD $=4.61)$, Pseudomonas ( $L S D=3.18)$, and Klebsiella (LSD = 3.34), which were consistent with the gut microbial communities' composition results. 
Moreover, five opportunistic pathogens were isolated from V1000_A sample (Table S2), which included Klebsiella aerogenes (NKU-Kae), Klebsiella pneumoniae (NKU-Kpn7), Klebsiella oxytoca (NKU-Kox6), Achromobacter xylosoxidans (NKU-Axy), and Pseudomonas aeruginosa (NKU-Pae). Table S3 represents the minimal inhibitory concentration (MIC) of these opportunistic pathogens. Data suggested that all the isolated opportunistic pathogens were resistant to vancomycin with MIC $₫ 256 \mathrm{mg} \mathrm{L}^{-1}$, as well as betalactam antibiotics including ampicillin (MIC $₫ 64-256 \mathrm{mg} \mathrm{L}^{-1}$ ) and amoxicillin (MIC $₫ 64-256 \mathrm{mg} \mathrm{L}^{-1}$ ), sulfonamides (sulfamethoxazole MIC $\mathbb{1 2 8} 2856 \mathrm{mg} \mathrm{L}^{-1}$ ) and MLSB (erythromycin MIC $₫ 8-64 \mathrm{mg} \mathrm{L}^{-1}$ ). However, Klebsiella oxytoca (NKU-Kox6), Achromobacter xylosoxidans (NKU-Axy) and Pseudomonas aeruginosa (NKU-Pae) showed the more comprehensive resistant properties to several kinds of antibiotics than Klebsiella aerogenes (NKU-Kae) and Klebsiella pneumoniae (NKU-Kpn7), including kanamycin, tetracycline, and streptomycin. Table S4 showed a total of nine ARGs detecting in these opportunistic pathogens. The results showed that all the opportunistic pathogens carried qnrs and bl2be_shv2, and except NKU-Kae, all others contained aac3iia, vang, arna, rosb, and ant2ia ARGs. Therefore, their intrinsic ARGs such as vang, aac3iia, sul1, and bl2be_shv2 might be attributed to high values of MICs to vancomycin, gentamicin, sulfamethoxazole, ampicillin, and amoxicillin. As shown in Table S5, a considerable variability in serum resistance for the opportunistic pathogens was observed, which constituted an important virulence trait that allowed these opportunistic pathogens to persist in vivo. And the survival of Pseudomonas aeruginosa (NKU-Pae) strain was significantly higher (12-54 times) than other strains with a virulence level of Grade 5.

\section{Correlation between microbial taxa and human disease- related pathways or ARGs}

Figure 5 showed the results of co-occurrence patterns between microbial taxa and human disease-related pathways. It can be seen that significantly increased bacteria after vancomycin exposure such as Achromobacter and Klebsiella were positively associated with almost all of those human disease-related pathways, and the decreased bacteria were negatively associated with these pathways. Specifically, the correlation coefficients of Achromobacter with colorectal cancer, viral myocarditis and toxoplasmosis were about $0.8(P<0.01)$ and the correlation coefficient of Klebsiella oxytoca with Staphylococcus aureus infection was $0.7(P<0.01)$.

The network analysis of co-occurrence patterns between microbial taxa and ARG subtypes was shown in Fig. 6. Interestingly, a very similar pattern of results was observed that a significantly decreased the bacteria was positively associated with the most ARGs. For example, the correlation coefficients of Bacteroides fragilis with bl2e_cepa aac6ie and tet32 were about $1.0(P<0.001)$, and the correlation coefficient of Parabacteroides with ermb was about $0.8(P<0.01)$. Only two significantly increased bacterial genera were positively associated with several ARGs. For example, the strong correlations were found in Klebsiella oxytoca with yidy/mdtl and tetc (about 0.9, $\mathrm{P}<0.01$ ), and in Pseudomonas with ermf and tetq (about $0.8, \mathrm{P}<0.05$ ).

\section{Discussion}




\section{Vancomycin exposure significantly increased the opportunistic pathogens}

The SHIME model was stably operated in this study because the predominant phyla of Bacteriodetes, Proteobacteria, Synergistetes, and Firmicutes in the gut microbiome was previously demonstrated by Yu's group [33]. These predominant phyla had also been observed in fecal samples from human and animals $[34,35]$. Moreover, the inconsistent bacterial phyla and genera were identified in different colon regions, providing further evidence that colon regions play an essential role in shaping the structure of gut microbiota [18].

Numerous in vivo studies have reported the similar phenomena as shown in our research that significantly decreased of Bacteroidetes and Firmicutes phyla and predominantly detected human intestinal microbiota from Proteobacteria phylum such as Pseudomonas and Klebsiella following vancomycin administration $[9,10,15]$. Some studies have also reported a drop in richness and alpha diversity and significant effect on beta diversity pursued by vancomycin exposure $[9,36]$. Achromobacter, Pseudomonas, and Klebsiella genera were all confirmed to increase after vancomycin exposure, although these bacteria are all considered commensal in the human gut, fecal carriage of these opportunistic pathogens was confirmed to be a severe risk factor for the infection [11, 12]. Achromobacter and Klebsiella intrinsically carry ARGs such as vang, aac3iia, sul1, and bl2be_shv2, which make them inherently resistant to vancomycin, aminoglycosides, sulfonamides, and beta-lactam antibiotics, consequently pose a significant therapeutic challenge [37]. Additionally, the multidrug resistant Pseudomonas aeruginosa with high virulence obtained from this research has also been well recorded in many previous research papers, indicating its immense threat to human health [38]. Some commensal microbiota in the human gut that decreased after vancomycin exposure such as Bacteroides fragilis and Parabacteroides are novel probiotics $[39,40]$, and the decrease of these probiotics may cause dysbiosis of gut microbiota, which would lead human health problem $[41,42]$. Therefore, the results of our research demonstrated a serious impact and a negative side-effect of vancomycin that enriched pathogens related to health problems, which should be considered as a fundamental aspect of the cost-benefit equation for an antibiotic prescription.

\section{Opportunistic pathogens associated human disease-related pathways increased by vancomycin exposure}

The significant increase in opportunistic pathogens associated human disease-related pathways and decrease in ARGs of human intestinal microbiota following vancomycin treatment exhibited in this study have not been reported in details yet. However, ampicillin had been found to enhance glucose tolerance, and promote the selection of antimicrobial resistance genes in intestinal microbiota using animal models [43]. Importantly, this study displayed a positive correlation between opportunistic pathogens (Klebsiella and Achromobacter) and human disease-related pathways, which suggested the bloom of these opportunistic pathogens caused by vancomycin treatment that might contribute to human diseases. For instance, some studies have indicated that Klebsiella was enriched in cancer and diabetes patients [44], 
and be related to various infectious diseases [45] and neurosurgery disease [14]. Furthermore, Achromobacter was also reported to be related to human diseases such as pneumonia [46], colitis [47], cancer [48], and cardiovascular diseases [13]. All of the findings, as mentioned above, were also linked with positive correlations between the abundances of these opportunistic pathogens and human diseaserelated pathways in our study. Some previous studies have also reported multiple ARGs resided in these types of pathogenic bacteria $[49,50]$, that maybe one of the critical reason for ARGs were more abundantly present in control samples.

\section{Vancomycin effects were "SHIME-compartment" different and persistent}

The phenomenon observed in the SHIME model that a larger shift of microbiota found in the ascending colon was also revealed by previous reporters. They demonstrated that changes of intestinal microbiota by vancomycin or mixed antibiotics exposure were "SHIME-compartment" different $[17,18]$. Meanwhile, more extensive shifts in bloomed opportunistic pathogens associated human disease-related pathways as well as ARGs found in this study were in line with other research that had clarified the primary effect observed at the level of the microbiota could also be found at genomic and metabolic levels [51, 52]. The intestinal microbiota is a key "organ" for the individual's health and their different shifting in the ascending colon and descending colon suggested that more studies should focus on the proximal colon for the uniform data sheets of colon microbiota. However, in vivo experiments usually analyses the feces that standing for the distal intestinal microbiota. Furthermore, the vancomycin effects were still evident for at least two weeks after the vancomycin discontinued, although the resilient reduction of opportunistic pathogens and human disease-related pathways was observed. Reijnders and colleagues revealed that a markedly decreased of microbial diversity, decreased relative abundance of most Grampositive bacteria of the Firmicutes, and increased Gram-negative Proteobacteria caused by vancomycin treatment persisted 56 days after cessation of antibiotic therapy [15]. Isaac and colleagues also reported a similar phenomenon, which revealed that 14 days treatment with vancomycin induced persistent reduction of the abundances of OTU13 and OTU23, and diminishment of richness till postnatal weeks 22 after antibiotic treatment discontinuation [9].

\section{FMT restored microbiome composition and genes}

Finally, this study revealed that the FMT would restore human intestinal microbiome composition, human disease-related pathways, and ARGs that profoundly affected by vancomycin. The restoration of microbiome composition find in our study may refer to the findings by Lee and associates that germ-free mice mono-associated with a single Bacteroides species are resistant to colonization by the same, but not different, species [53]. FMT may also colonize missing flora after vancomycin exposure with priority to restore the dysbiosis. For recurrent of Clostridium difficile infection, several researchers have suggested that FMT is a superior method to oral vancomycin for the treatment [21,22], and combinations of them would be a better choice attributed to restore the intestinal microbiota [23,24]. The clinical application of FMT also includes inflammatory bowel disease, diabetes mellitus, liver cirrhosis, gut-brain disease, and others [54]. Moreover, the FMT has become a completely novel way to eradicate drug-resistant bacteria 
from the intestinal reservoir, which would include decolonization of extended-spectrum beta-lactamase (ESBL)-producing and carbapenemase-producing Enterobacteriaceae, vancomycin-resistant Enterococci, or methicillin-resistant Staphylococcus aureus [55]. However, the clinical utility of FMT to displace the multidrug-resistant bacteria or treat patients with ulcerative colitis (UC) is still controversial because of little benefit shown in other papers, and the use of probiotics maybe better with potentially lower risks of adverse events [56-59]. Thus, there would be much work to reveal whether FMT will be promising in clinical application that limit opportunistic pathogens overgrowth and restore human intestinal microbiota composition and genes that profound affected by antibiotic exposure.

\section{Perspectives}

Typical antibiotics have been detected in the collective gut of the Chinese population in our previous research which provided a reference for this study of the effects of vancomycin on human gut microbiota [4]. However, there were several limitations of this study. First, repeating these experiments at least a number of times more would be better for currently stating that vancomycin causes a significant disruption on gut microbiota and confirming the benefit of FMT on vancomycin induced dysbiosis, which were not taken attributed to the costs associated with system operation and samples detection. On the other hand, the comparation of opportunistic pathogens isolated from control sample with vancomycin treated sample will also be necessary to reveal its effect in bacteria characteristic. It is also of interest to inoculate the microbiota mixture from several healthy humans as well as patient to discover the different effects of vancomycin between individuals. Further in vivo studies are also needed to verify whether the findings in this in vitro study reflect the real recovery in vivo. Moreover, research on the restoration effects of some prebiotics, probiotics, and synbiotics during and after antibiotic therapy will also be promising, which may help discover several clinical strategies to restore side effects caused by antibiotics. As the increasing of opportunistic pathogens and human disease-related genes by antibiotics exposure, which may pose a significant therapeutic challenge and take some efficient measures to reduce or even eliminate the effects after antibiotic treatment should be more critical.

\section{Conclusions}

Exposure to vancomycin significantly altered the taxonomic composition and diversity, and it subsequently increased opportunistic pathogens and human disease-related pathways. The changes were "SHIME-compartment" different with a more significant effect on the ascending colon. Moreover, bacteria that increased after vancomycin exposure were positively associated with the human diseaserelated pathways, while bacterial decreased were positively related to quantified ARGs. The significant shifted human gut microbiota was not recovered after two weeks of vancomycin discontinuance; however, restored thoroughly after FMT. These results may be valuable for directing future work, which may open up new perspectives for assessing the direct effects of antibiotics on the intestinal microbiota and suggested that FMT may be promising in clinical application.

\section{Declarations}




\section{Acknowledgments}

The authors express the sincerest thanks to Professor Bing Wu (School of the Environment in Nanjing University, Nanjing in China) for the guidance of SHIME and Guangzhou Gene Denovo Co., Ltd and Anhui MicroAnaly Gene Technologies Co., Ltd for genome sequencing and analysis.

\section{Authors' contributions}

LL carried out the laboratory work and wrote the manuscript. QW revised this manuscript. DR edited the language and improved the clarity of this manuscript. HQ, HL, JS, SW and JY carried out the laboratory work. YX provided funding support. DM and YL revised this manuscript. All authors read and approved the final manuscript.

\section{Funding}

This work was supported by the Key projects of the National Natural Science Foundation of China (41831287), China National Funds for Distinguished Young Scientists (41525013), National Natural Science Foundation of China $(31870351,41703088,31670509$, and 21607016), Key projects of Research and Development of Hebei Province (19273707D).

\section{Availability of data and materials}

Please contact the author for data requests.

\section{Ethics approval and consent to participate}

The study was approved by the Biomedical Ethics Committees of Nankai University. The participant has given written informed consent to understand the study purpose, procedures, risks, benefits, and rights.

\section{Consent for publication}

Not applicable.

\section{Competing interests}

The authors declare that they have no competing interests.

\section{Author details}

liulei19880114@qq.com (L. Liu)

wangqing@hebeu.edu.cn (Q. Wang)

898975347@qq.com (H. Qi)

anmicro_85@yahoo.co.in (R. Das) 
sjl_net@163.com (J.Shi)

15330856579@163.com (S. Wang)

yangjing1@sust.edu.cn (J. Yang)

zxyg@126.com (Y. Xue)

maodq@nankai.edu.cn (D. Mao)

luoy@nankai.edu.cn (Y. Luo)

\section{Abbreviations}

ARGs: Antibiotic resistance genes; ESBL: Extended-spectrum beta-lactamase; FMT: Fecal microbiota transplantation; HT-qPCR: High-throughput quantitative PCR; LB: Luria-Bertani; LEfSe: Linear discriminant analysis effect size; MGEs: Mobile genetic elements; MIC: Minimal inhibitory concentration; NMDS: Nonmetric multidimensional scaling; PCoA: Principal coordinate analysis; SHIME: Simulator of the human intestinal microbial ecosystem; PCR: Polymerase chain reaction; PICRUSt: Phylogenetic Investigation of Communities by Reconstruction of Unobserved States; QIIME: Quantitative Insights Into Microbial Ecology; RDP: Ribosomal Database Project; SRA: Sequence Read Archive; UC: Ulcerative colitis

\section{References}

1. Khan I, Yasir M, Azhar El, Kumosani T, Barbour EK, Bibi F, Kamal MA: Implication of gut microbiota in human health. CNS Neurol Disord Drug Targets 2014, 13(8):1325-1333

2. Cabreiro F, Au C, Leung KY, Vergara-Irigaray N, Cocheme HM, Noori T, Weinkove D, Schuster E, Greene ND, Gems D: Metformin retards aging in $C$. elegans by altering microbial folate and methionine metabolism. Cell 2013, 153(1):228-239.

3. Qin JJ, Li RQ, Raes J, Arumugam M, Burgdorf KS, Manichanh C, Nielsen T, Pons N, Levenez F, Yamada $\mathrm{T}$ et al: A human gut microbial gene catalogue established by metagenomic sequencing. Nature 2010, 464(7285):59-65.

4. Wang Q, Duan YJ, Wang SP, Wang LT, Hou ZL, Cui YX, Hou J, Das R, Mao DQ, Luo Y: Occurrence and distribution of clinical and veterinary antibiotics in the faeces of a Chinese population. $J$ Hazard Mater 2020, 383:121129.

5. Bengtsson-Palme J, Kristiansson E, Larsson DGJ: Environmental factors influencing the development and spread of antibiotic resistance. FEMS Microbiol Rev 2018, 42(1):68-80.

6. Stecher B, Maier L, Hardt WD: 'Blooming' in the gut: how dysbiosis might contribute to pathogen evolution. Nat Rev Microbiol 2013, 11(4):277-284. 
7. Blaser MJ: Antibiotic use and its consequences for the normal microbiome. Science 2016, 352(6285):544-545.

8. laniro G, Tilg H, Gasbarrini A: Antibiotics as deep modulators of gut microbiota: between good and evil. Gut 2016, 65(11):1906-1915.

9. Isaac S, Scher JU, Djukovic A, Jimenez N, Littman DR, Abramson SB, Pamer EG, Ubeda C: Short- and long-term effects of oral vancomycin on the human intestinal microbiota. $J$ Antimicrob Chemother 2017, 72(1):128-136.

10. Vrieze A, Out C, Fuentes S, Jonker L, Reuling I, Kootte RS, van Nood E, Holleman F, Knaapen M, Romijn JA et al: Impact of oral vancomycin on gut microbiota, bile acid metabolism, and insulin sensitivity. J Hepatol 2014, 60(4):824-831.

11. Gorrie C, Mirceta M, Wick R, Edwards D, Thomson N, Strugnell R, Pratt N, Garlick J, Watson K, Pilcher $\mathrm{D}$ et al: Gastrointestinal carriage is a major reservoir of Klebsiella pneumoniae infection in intensive care patients. Clin Infect Dis 2017, 65(2):208-215.

12. Kwon $Y$, Koene RJ, Cross $C$, McEntee J, Green JS: Fatal non-thrombotic pulmonary embolization in a patient with undiagnosed factitious disorder. BMC Res Notes 2015, 8:302.

13. Rodrigues CG, Rays J, Kanegae MY: Native-valve endocarditis caused by Achromobacter xylosoxidans. a case report and review of literature. Autops Case Rep 2017, 7(3):50-55.

14. Shen Y, Xu J, Li Z, Huang Y, Yuan Y, Wang J, Zhang M, Hu S, Liang Y: Analysis of gut microbiota diversity and auxiliary diagnosis as a biomarker in patients with schizophrenia: a cross-sectional study. Schizophr Res 2018, 197:470-477.

15. Reijnders D, Goossens GH, Hermes GD, Neis EP, van der Beek CM, Most J, Holst JJ, Lenaerts K, Kootte RS, Nieuwdorp $M$ et al: Effects of gut microbiota manipulation by antibiotics on host metabolism in obese humans: a randomized double-blind placebo-controlled trial. Cell Metab 2016, 24(1):63-74.

16. Marzorati $\mathrm{M}$, Van de Wiele T: An advanced in vitro technology platform to study the mechanism of action of prebiotics and probiotics in the gastrointestinal tract. J Clin Gastroentero/ 2016, 50:S124S125.

17. Ichim TE, Kesari S, Shafer K: Protection from chemotherapy- and antibiotic-mediated dysbiosis of the gut microbiota by a probiotic with digestive enzymes supplement. Oncotarget 2018, 9(56):3091930935.

18. Marzorati M, Vilchez-Vargas R, Bussche JV, Truchado P, Jauregui R, El Hage RA, Pieper DH, Vanhaecke L, Van de Wiele T: High-fiber and high-protein diets shape different gut microbial communities, which ecologically behave similarly under stress conditions, as shown in a gastrointestinal simulator. Mol Nutr Food Res 2017, 61(1):1600150.

19. Van den Abbeele P, Roos S, Eeckhaut V, MacKenzie DA, Derde M, Verstraete W, Marzorati M, Possemiers S, Vanhoecke B, Van Immerseel F et al: Incorporating a mucosal environment in a dynamic gut model results in a more representative colonization by Lactobacilli. Microb Biotechnol 2012, 5(1):106-115. 
20. El Hage R, Hernandez-Sanabria E, Calatayud Arroyo M, Props R, Van de Wiele T: Propionateproducing consortium restores antibiotic-induced dysbiosis in a dynamic in vitro model of the human intestinal microbial ecosystem. Front Microbiol 2019, 10:1206.

21. Lubbert C, Salzberger B, Mossner J: Fecal microbiota transplantation. Internist 2017, 58(5):456-468.

22. Khan MY, Dirweesh A, Khurshid T, Siddiqui WJ: Comparing fecal microbiota transplantation to standard-of-care treatment for recurrent Clostridium difficile infection: a systematic review and metaanalysis. Eur J Gastroenterol Hepatol 2018, 30(11):1309-1317.

23. Bulow C, Langdon A, Hink T, Wallace M, Reske KA, Patel S, Sun X, Seiler S, Jones S, Kwon JH et al: Impact of amoxicillin-clavulanate followed by autologous fecal microbiota transplantation on fecal microbiome structure and metabolic potential. mSphere 2018, 3(6):e00588-00518.

24. Hota SS, Sales V, Tomlinson G, Salpeter MJ, McGeer A, Coburn B, Guttman DS, Low DE, Poutanen SM: Oral vancomycin followed by fecal transplantation versus tapering oral vancomycin treatment for recurrent Clostridium difficile infection: an open-label, randomized controlled trial. Clin Infect Dis 2017, 64(3):265-271.

25. Van den Abbeele P, Belzer C, Goossens M, Kleerebezem M, De Vos WM, Thas O, De Weirdt R, Kerckhof FM, Van de Wiele T: Butyrate-producing Clostridium cluster XIVa species specifically colonize mucins in an in vitro gut model. Isme $J 2013,7(5): 949-961$.

26. Caporaso JG, Kuczynski J, Stombaugh J, Bittinger K, Bushman FD, Costello EK, Fierer N, Pena AG, Goodrich JK, Gordon JI et al: QIIME allows analysis of high-throughput community sequencing data. Nat Methods 2010, 7(5):335-336.

27. Wang Q, Garrity GM, Tiedje JM, Cole JR: Naive Bayesian classifier for rapid assignment of rRNA sequences into the new bacterial taxonomy. App/ Environ Microbiol 2007, 73(16):5261-5267.

28. Lozupone $C$, Knight R: UniFrac: a new phylogenetic method for comparing microbial communities. Appl Environ Microbiol 2005, 71(12):8228-8235.

29. Segata N, Izard J, Waldron L, Gevers D, Miropolsky L, Garrett WS, Huttenhower C: Metagenomic biomarker discovery and explanation. Genome Bio/ 2011, 12(6):R60.

30. Langille MGI, Zaneveld J, Caporaso JG, McDonald D, Knights D, Reyes JA, Clemente JC, Burkepile DE, Thurber RLV, Knight $R$ et al: Predictive functional profiling of microbial communities using 16S rRNA marker gene sequences. Nat Biotechnol 2013, 31(9):814-821.

31. Hu Y, Yang X, Qin J, Lu N, Cheng G, Wu N, Pan Y, Li J, Zhu L, Wang X et al: Metagenome-wide analysis of antibiotic resistance genes in a large cohort of human gut microbiota. Nat Commun 2013, 4:2151-2249.

32. Rosen DA, Hilliard JK, Tiemann KM, Todd EM, Morley SC, Hunstad DA: Klebsiella pneumoniae FimK promotes virulence in murine pneumonia. J Infect Dis 2016, 213(4):649-658.

33. Yu HY, Wu B, Zhang XX, Liu S, Yu J, Cheng SP, Ren HQ, Ye L: Arsenic metabolism and toxicity influenced by ferric iron in simulated gastrointestinal tract and the roles of gut microbiota. Environ Sci Technol 2016, 50(13):7189-7197. 
34. Gao Z, Guo B, Gao R, Zhu Q, Qin H: Microbiota disbiosis is associated with colorectal cancer. Front Microbiol 2015, 6:1-9.

35. Li S, Zhang C, Gu Y, Chen L, Ou S, Wang Y, Peng X: Lean rats gained more body weight than obese ones from a high-fibre diet. Br J Nutr 2015, 114(8):1188-1194.

36. Lankelma JM, Cranendonk DR, Belzer C, de Vos AF, de Vos WM, van der Poll T, Wiersinga WJ: Antibiotic-induced gut microbiota disruption during human endotoxemia: a randomised controlled study. Gut 2017, 66(9):1623-1630.

37. Munoz-Price LS, Poirel L, Bonomo RA, Schwaber MJ, Daikos GL, Cormican M, Cornaglia G, Garau J, Gniadkowski M, Hayden MK et al: Clinical epidemiology of the global expansion of Klebsiella pneumoniae carbapenemases. Lancet Infect Dis 2013, 13(9):785-796.

38. Chen J, Chen Y, Hu P, Zhou T, Xu X, Pei X: Risk assessment of infected children with Pseudomonas aeruginosa pneumonia by combining host and pathogen predictors. Infect Genet Evol 2018, 57:8287.

39. Wang Y, Deng H, Li Z, Tan Y, Han Y, Wang X, Du Z, Liu Y, Yang R, Bai Y et al: Safety evaluation of a novel strain of Bacteroides fragilis. Front Microbiol 2017, 8:435.

40. Wu TR, Lin CS, Chang CJ, Lin TL, Martel J, Ko YF, Ojcius DM, Lu CC, Young JD, Lai HC: Gut commensal Parabacteroides goldsteinii plays a predominant role in the anti-obesity effects of polysaccharides isolated from Hirsutella sinensis. Gut 2019, 68(2):248-262.

41. George Kerry R, Patra JK, Gouda S, Park Y, Shin HS, Das G: Benefaction of probiotics for human health: a review. J Food Drug Anal 2018, 26(3):927-939.

42. Wischmeyer PE, McDonald D, Knight R: Role of the microbiome, probiotics, and 'dysbiosis therapy' in critical illness. Curr Opin Crit Care 2016, 22(4):347-353.

43. Membrez M, Blancher F, Jaquet M, Bibiloni R, Cani PD, Burcelin RG, Corthesy I, Mace K, Chou CJ: Gut microbiota modulation with norfoxacin and ampicillin enhances glucose tolerance in mice. FASEB $J$ 2008, 22(7):2416-2426.

44. Zhang Y, Zhao C, Wang Q, Wang X, Chen H, Li H, Zhang F, Li S, Wang R, Wang H: High prevalence of hypervirulent Klebsiella pneumoniae infection in China: geographic distribution, clinical characteristics, and antimicrobial resistance. Antimicrob Agents Chemother 2016, 60(10):6115-6120.

45. Boix-Palop L, Xercavins M, Badía C, Obradors M, Riera M, Freixas N, Pérez J, Rodríguez-Carballeira M, Garau J, Calbo E: Emerging extended-spectrum $\beta$-lactamase-producing Klebsiella pneumoniae causing community-onset urinary tract infections: a case-control-control study. Int J Antimicrob Agents 2017, 50(2):197-202.

46. Liu C, Pan F, Guo J, Yan W, Jin Y, Liu C, Qin L, Fang X: Hospital acquired pneumonia due to Achromobacter spp. in a geriatric ward in China: clinical characteristic, genome variability, biofilm production, antibiotic resistance and integron in isolated strains. Front Microbio/ 2016, 7:621.

47. Walujkar SA, Kumbhare SV, Marathe NP, Patangia DV, Lawate PS, Bharadwaj RS, Shouche YS: Molecular profiling of mucosal tissue associated microbiota in patients manifesting acute exacerbations and remission stage of ulcerative colitis. World J Microbiol Biotechnol 2018, 34(6):76. 
48. Kynaston K, Ho M, Sánchez-Vargas F, Paruolo J, Stutzman T, Velez A, Greene J: Achromobacter xylosoxidans bacteremia in patients with cancer. Open Forum Infect Di 2015, 2(suppl_1):479-484.

49. Do TT, Tamames J, Stedtfeld RD, Guo X, Murphy S, Tiedje JM, Walsh F: Antibiotic resistance gene detection in the microbiome context. Microb Drug Resist 2018, 24(5):542-546.

50. Justesen US, Acar Z, Sydenham TV, Johansson A, Esgai: Antimicrobial susceptibility testing of Bacteroides fragilis using the MALDI Biotyper antibiotic susceptibility test rapid assay (MBT-ASTRA). Anaerobe 2018, 54(SI):236-239.

51. Garcia-Villalba R, Vissenaekens H, Pitart J, Romo-Vaquero M, Espin JC, Grootaert C, Selma MV, Raes K, Smagghe G, Possemiers S et al: Gastrointestinal simulation model TWIN-SHIME shows differences between human urolithin-metabotypes in gut microbiota composition, pomegranate polyphenol metabolism, and transport along the Intestinal tract. J Agric Food Chem 2017, 65(27):5480-5493.

52. Wang Y, Rui M, Nie Y, Lu G: Influence of gastrointestinal tract on metabolism of bisphenol $A$ as determined by in vitro simulated system. J Hazard Mater 2018, 355:111-118.

53. Lee SM, Donaldson GP, Mikulski Z, Boyajian S, Ley K, Mazmanian SK: Bacterial colonization factors control specificity and stability of the gut microbiota. Nature 2013, 501(7467):426-429.

54. Zhang FM, Cui BT, He XX, Nie YQ, Wu KC, Fan DM, Grp F-SS: Microbiota transplantation: concept, methodology and strategy for its modernization. Protein Cel/ 2018, 9(5):462-473.

55. Manges AR, Steiner TS, Wright AJ: Fecal microbiota transplantation for the intestinal decolonization of extensively antimicrobial-resistant opportunistic pathogens: a review. Infect Dis 2016, 48(8):587592.

56. Hempel S, Newberry SJ, Maher AR, Wang Z, Miles JN, Shanman R, Johnsen B, Shekelle PG: Probiotics for the prevention and treatment of antibiotic-associated diarrhea: a systematic review and meta-analysis. JAMA 2012, 307(18):1959-1969.

57. Nishida A, Imaeda H, Ohno M, Inatomi O, Bamba S, Sugimoto M, Andoh A: Efficacy and safety of single fecal microbiota transplantation for Japanese patients with mild to moderately active ulcerative colitis. J Gastroenterol 2017, 52(4):476-482.

58. Vila J: Microbiota transplantation and/or CRISPR/Cas in the battle against antimicrobial resistance. Clin Microbiol Infect 2018, 24(7):684-686.

59. Huttner BD, de Lastours V, Wassenberg M, Maharshak N, Mauris A, Galperine T, Zanichelli V, Kapel N, Bellanger A, Olearo $\mathrm{F}$ et al: A 5-day course of oral antibiotics followed by faecal transplantation to eradicate carriage of multidrug-resistant Enterobacteriaceae. a randomized clinical trial. Clin Microbiol Infect 2019, 25(7):830-838.

\section{Figures}




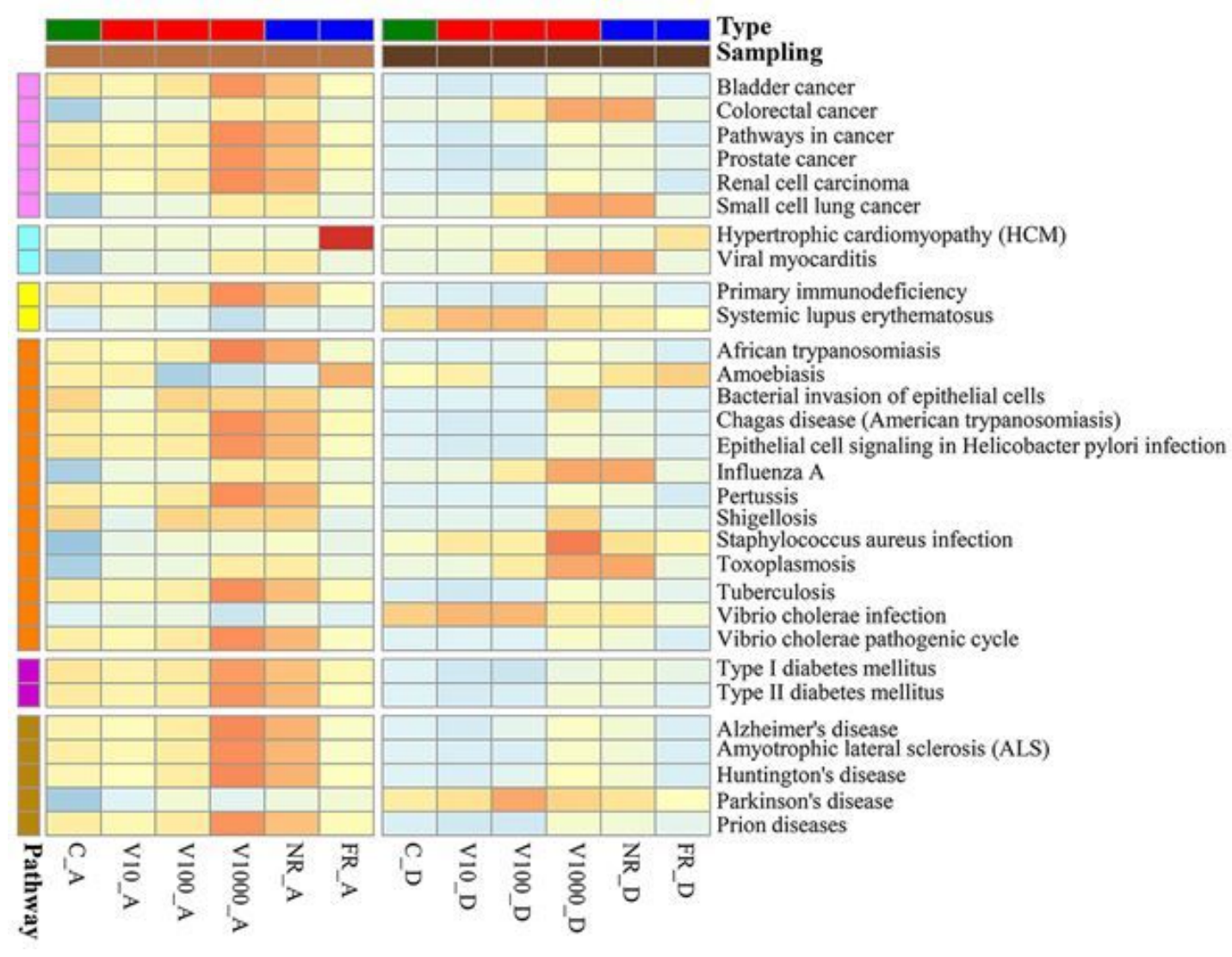

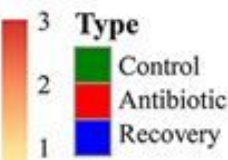

Sampling

0 Ascending colon

-1 Descending colon

Pathway

-2 Cancers

-3 Cardiovascular diseases Immune system diseases Infectious diseases

Metabolic diseases

Neurodegenerative diseases

\section{Figure 2}

Heatmap of human disease-related pathways in the two parts of colon with different treatment. Heatmap colors reflect gene numbers of human disease-related pathways from low (blue) to high (red). 


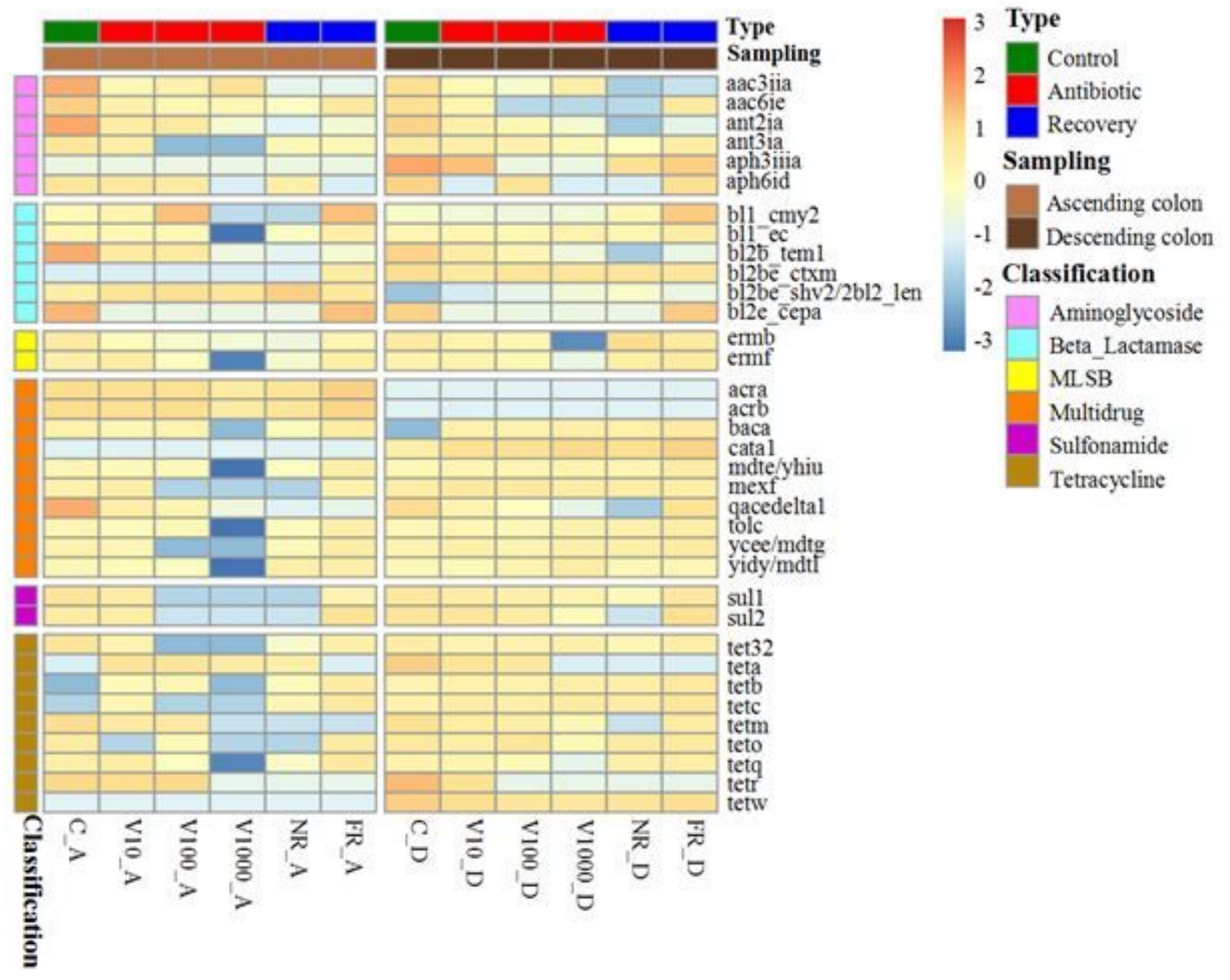

Figure 4

Heatmap of antibiotic resistance genes (ARGs) in the two parts of colon with different treatment. Heatmap colors reflect relative abundance of ARGs from low (blue) to high (red). 
(a)

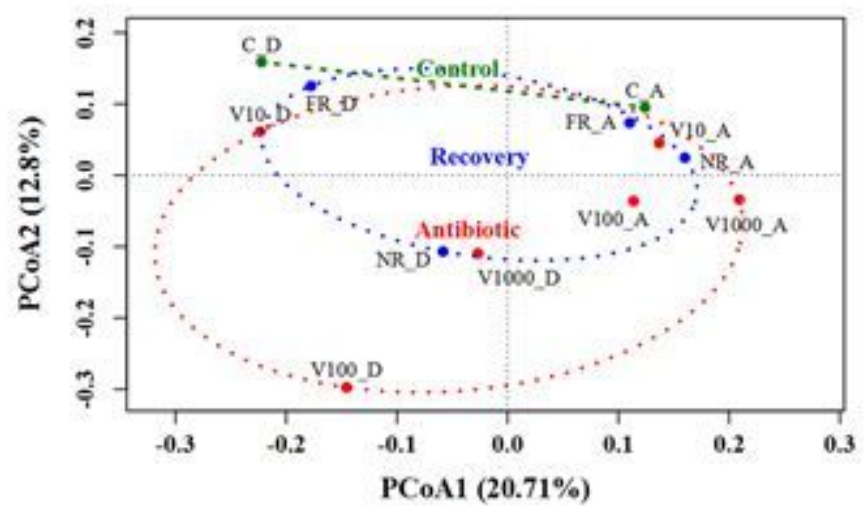

(c)

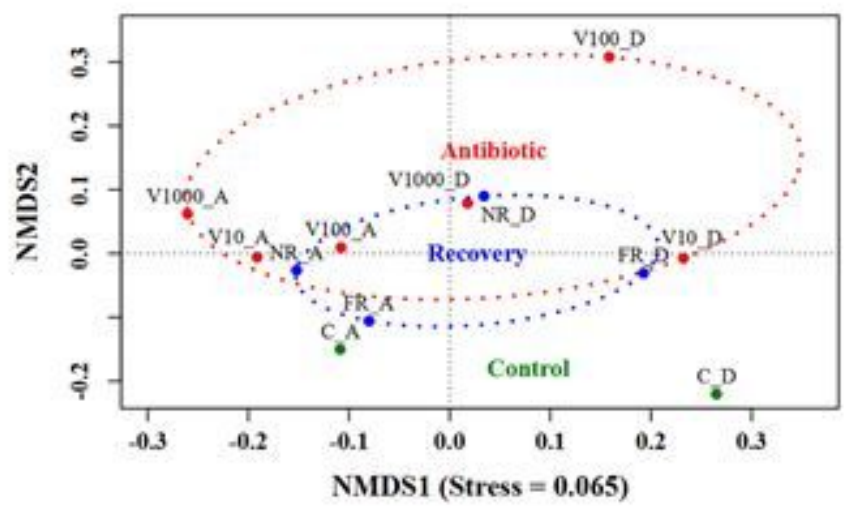

(b)

Weighted PCoA analysis in different groups

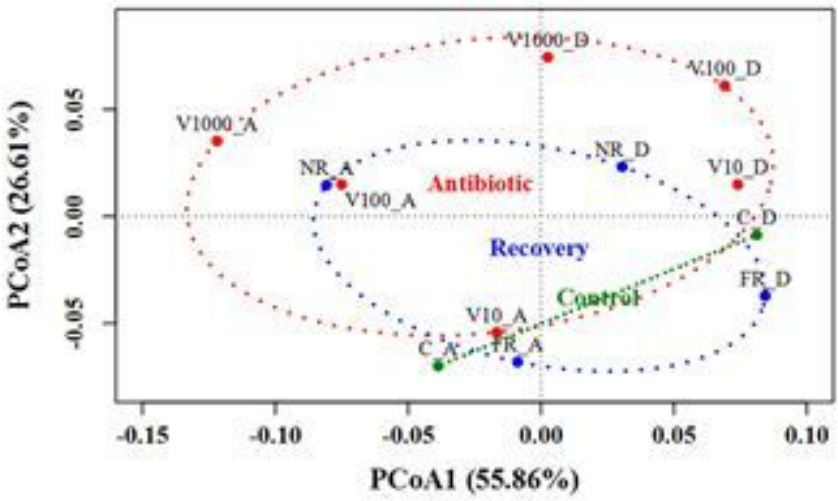

(d)

\section{Weighted NMDS analysis in different groups}

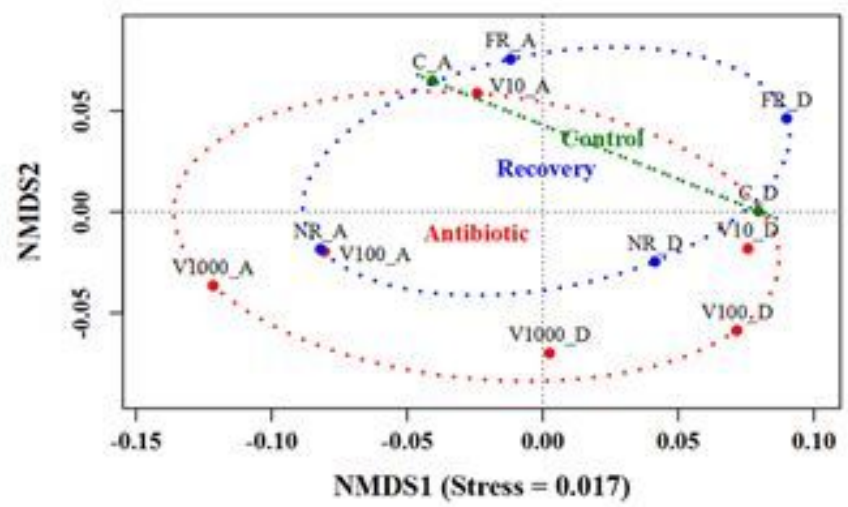

Figure 6

Effect of vancomycin treatment on gut microbiota beta diversity of the control (green), vancomycin (red), and recovery (blue) groups. Unweighted (a) and weighted (b) PCoA, and unweighted (c) and weighted (d) NMDS of UniFrac distances of samples in six groups (C, V10, V100, V1000, NR and FR). 
(a)

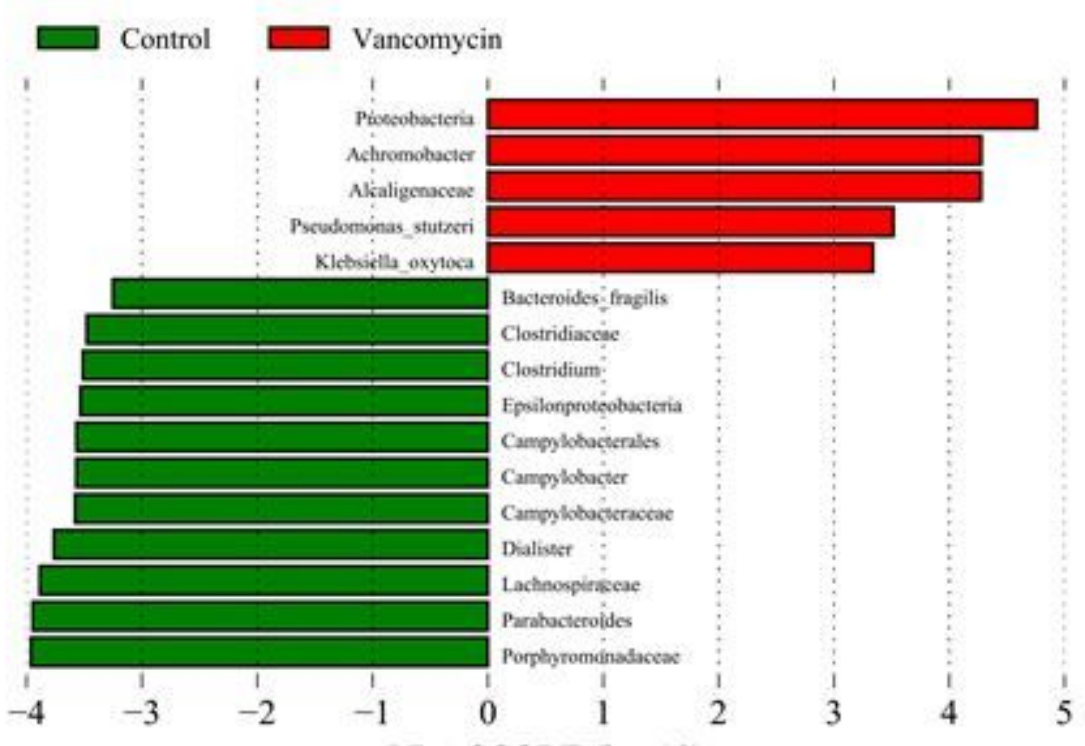

(b)

LDA SCORE $(\log 10)$

Cladogram

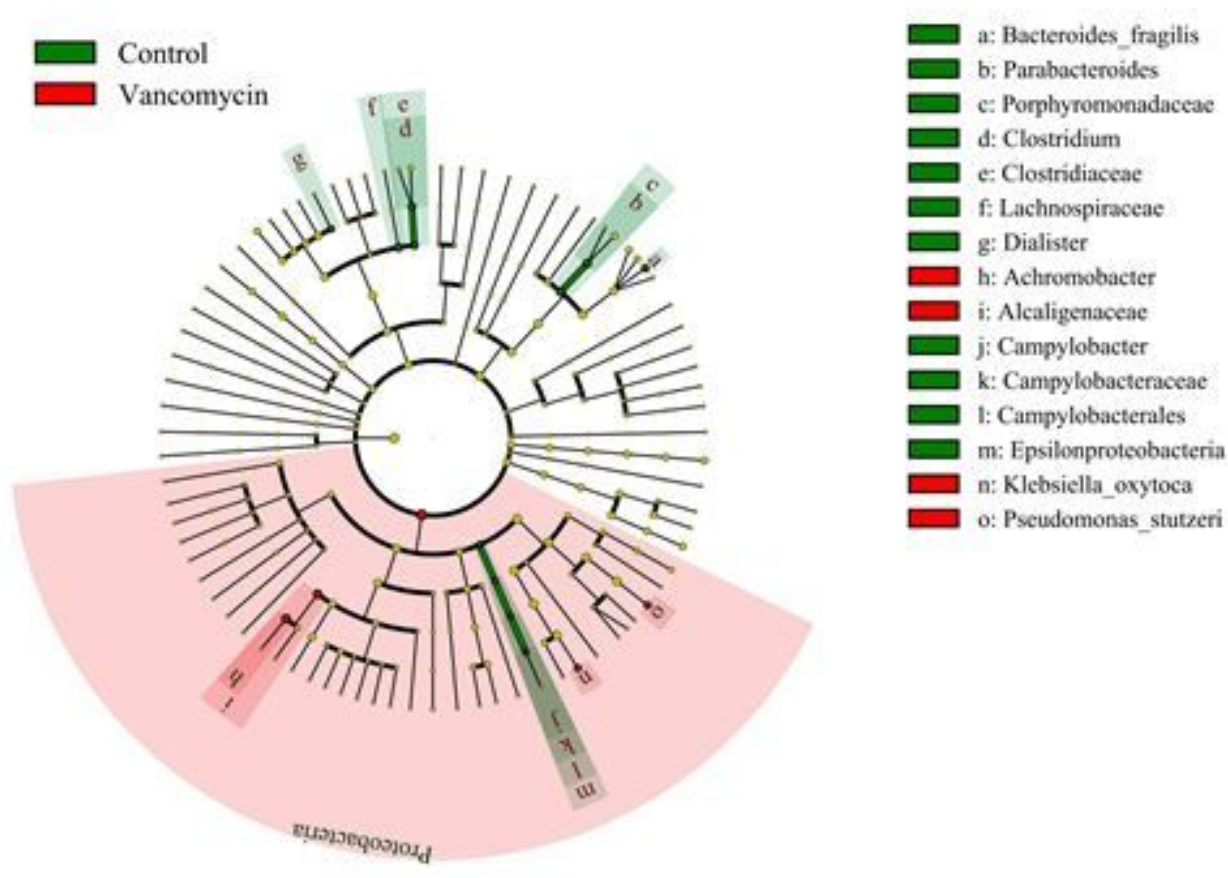

Figure 8

LDA score (a) and cladogram of LEfSe (b) comparison analysis between the control and antibiotic groups. The red, green shading depicts bacterial taxa that were significantly higher in either the control or antibiotic groups, as indicated. Selection of discriminative taxa between the control and antibiotic groups were based on an LDA score cutoff of 3.0, and differences in the relative abundances of taxa were statistically determined based on a Mann-Whitney test at a significance level of 0.05 . 


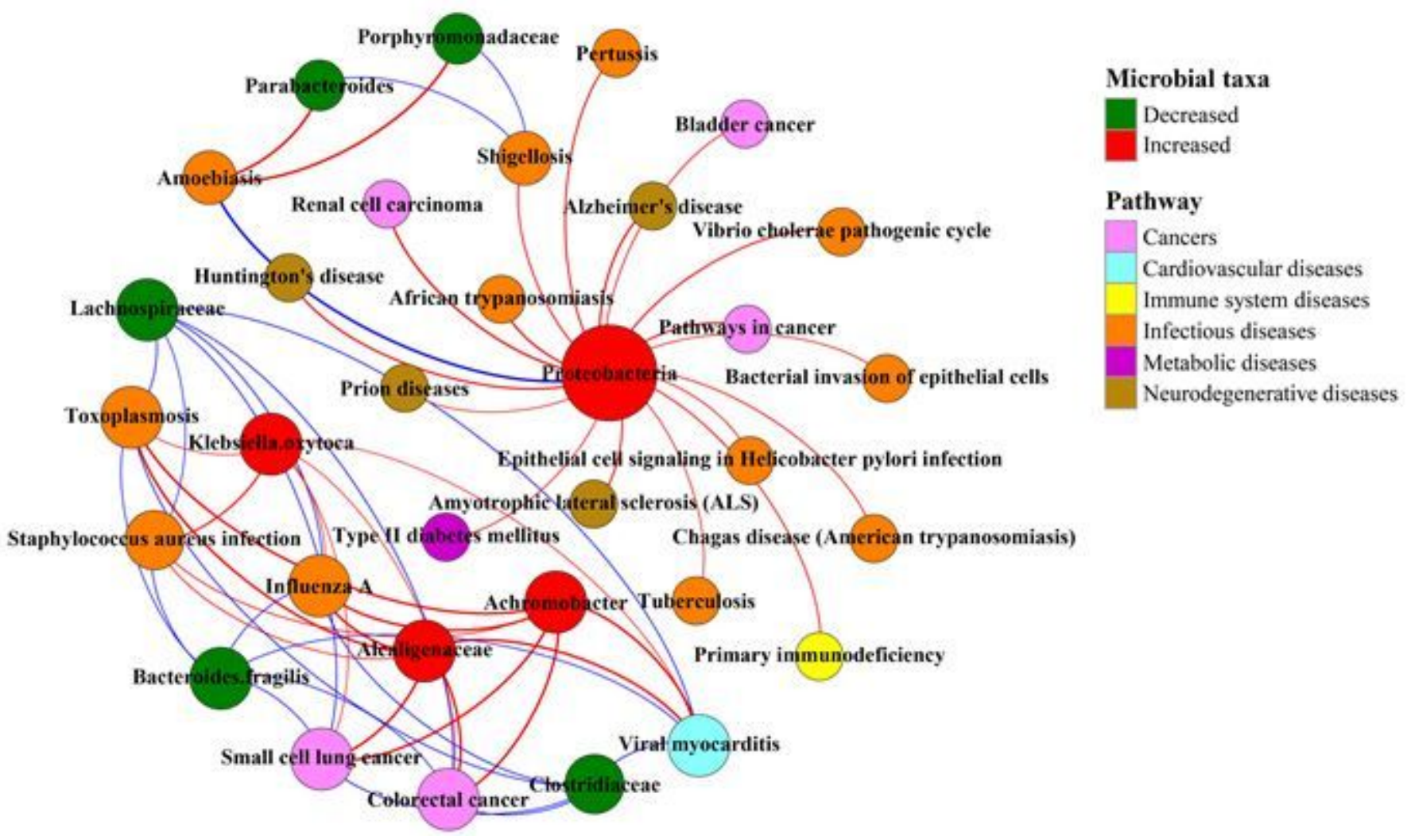

Figure 10

Network analysis revealing the co-occurrence patterns between microbial taxa and human disease-related pathways. The nodes in Network were colored according to types of human disease-related pathways and microbial genera that increased (red) or decreased (green) after vancomycin exposure. The edges were colored according to positive (red) or negative (blue) correlation. A connection represents strong and significant ( $P$ value $<0.05, r>0.6$ ) correlation. The size of each node is proportional to the number of connections, that is, degree. 


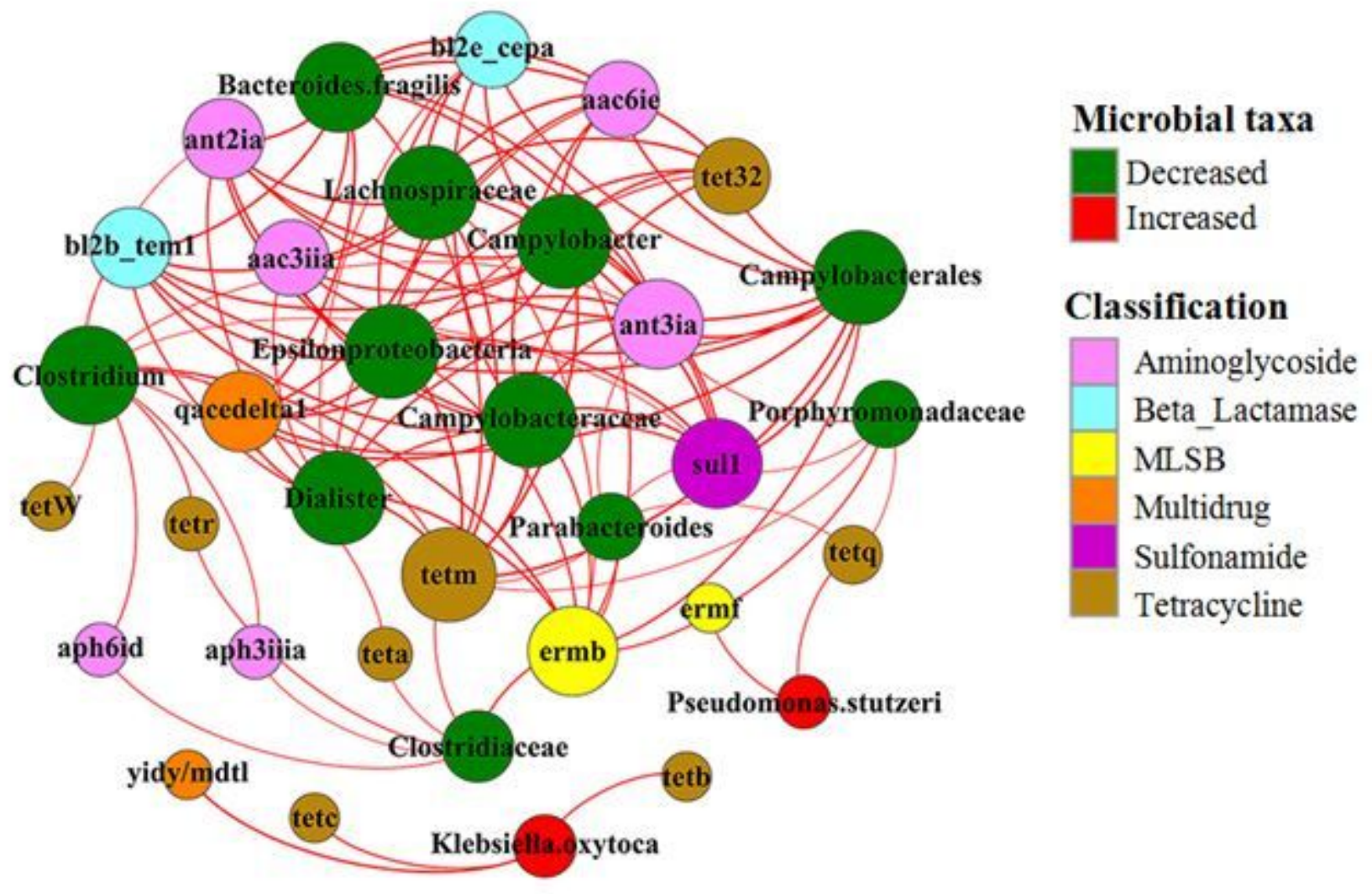

\section{Figure 11}

Network analysis revealing the co-occurrence patterns between microbial taxa and ARG subtypes. The nodes in Network were colored according to ARG types and microbial genera that increased (red) or decreased (green) after vancomycin exposure. The edges were colored according to positive (red) or negative (blue) correlation. A connection represents strong and significant ( $P$ value $<0.05, r>0.6)$ correlation. The size of each node is proportional to the number of connections, that is, degree.

\section{Supplementary Files}

This is a list of supplementary files associated with this preprint. Click to download.

- Additionalfile1.pdf

- Additionalfile2.xIsx

- Additionalfile1.pdf

- Additionalfile2.xlsx 